\title{
WILEY-VCH
}

DOI: 10.1002/ ((please add manuscript number))

Article type: Full Paper

\section{Metaparticles: dressing nano-objects with a hyperbolic coating}

Pan Wang*, Alexey V. Krasavin, Francesco N. Viscomi, Ali M. Adawi, Jean-Sebastien Bouillard, Lei Zhang, Diane J. Roth, Limin Tong, Anatoly V. Zayats*

Dr. P. Wang, Dr. A. V. Krasavin, Dr. J. -S. Bouillard, Dr. D. J. Roth, Prof. A. V. Zayats

Department of Physics, King's College London, Strand, London WC2R 2LS, UK

E-mail: pan.wang@kcl.ac.uk, a.zayats@kcl.ac.uk

F. N. Viscomi, Dr. A. M. Adawi, Dr. J. -S. Bouillard

School of Mathematics and Physical Sciences, University of Hull, Cottingham Road, Hull, HU6 7RX, UK

Dr. J. -S. Bouillard

G. W. Gray Centre for Advanced Materials, Cottingham Road, Hull, HU6 7RX, UK

Prof. L. Zhang, Prof. L. M. Tong

State Key Laboratory of Modern Optical Instrumentation, College of Optical Science and Engineering, Zhejiang University, Hangzhou 310027, China

Keywords: metaparticles, hyperbolic metamaterials, plasmonics, multishells, coating

The ability to engineer the optical response of a plasmonic nano-object is highly desired to achieve better control over light-matter interactions. Due to the sensitivity of plasmon resonances to the surrounding media, isotropic dielectric coating is an easy approach to modify the optical properties of a plasmonic nanostructure. However, the choice of coatings and the provided tunability is limited by the range of refractive indices of available materials. Here, we show that coating of plasmonic nano-objects with an anisotropic metamaterial, which displays a hyperbolic dispersion and allows the design of refractive index on demand, provides greater flexibility to engineer their interaction with light. This is experimentally demonstrated by coating $\mathrm{Au}$ nanospheres with alternating $\mathrm{SiO}_{2}$ and $\mathrm{Au}$ multishells, which creates rich and highly tunable plasmonic modal structures covering a broad wavelength range $(\sim 400-2200 \mathrm{~nm})$ and produces high local field intensity enhancement ( $\sim 500$ fold $)$. The concept is extended to hyperbolic coating of dielectric nano-objects, confirming the nature of the modes to be related to the resonances in the hyperbolic layer. The implemented approach using coating with an engineered effective refractive index may find applications in plasmon-enhanced spectroscopy, nanolasers, nonlinearity design, photothermal conversions, and hot-electron generation. 


\section{WILEY-VCH}

\section{Introduction}

Localized surface plasmons are collective oscillations of a free electron gas in nanostructures, which have the ability to confine electromagnetic fields at a deep-subwavelength scale, produce high local-field enhancement and generate hot electrons. ${ }^{[1-3]}$ In general, it is highly desirable that the optical properties such as scattering, absorption or local-field distribution of a plasmonic nano-object can be engineered in the required spectral range and with required values in order to achieve better performance in various applications. ${ }^{[4-10]}$ For example, for plasmonenhanced spectroscopies ${ }^{[4,5]}$ or nonlinear optics, ${ }^{[6]}$ the ability to tune resonance peaks and achieve strong local-field enhancement is of vital importance. In plasmon-based optical sensing, the detection limit is determined by the local fields and resonance linewidth. ${ }^{[7,8]}$ At the same time, for energy harvesting, e.g., in photothermal conversion and photovoltaics, strong and broadband absorption is an important factor to be considered. ${ }^{[9,10]}$ In the past, great efforts have been devoted to engineering the optical properties of plasmonic nano-objects. ${ }^{[2,11-19]}$ These include tuning the plasmonic resonance of metal nanoparticles via material and morphology control, ${ }^{[11,13,14]}$ patterning metal nanoparticles into complex coupled structures to engineer collective plasmonic modes, ${ }^{[15,16]}$ using particle-on-a-mirror structures to greatly enhance the local fields, ${ }^{[2,17]}$ or coupling modes in a metal nanoparticle with that in a photonic cavity for spectral narrowing. ${ }^{[18,19]}$ Coating a plasmonic nano-object with an isotropic dielectric medium (Figure 1a,b) is another way to modify the optical properties, ${ }^{[20]}$ which does not require precise and complex positioning, and can be easily realized in a scalable manner. However, due to the limited range of refractive indices available in conventional materials, the ability to create an essentially modified and enriched modal structure using this method is rather limited. Instead, such coating is commonly used for the stabilization of nanoparticles or as a dielectric spacer to isolate nanoparticles from their surroundings.

Electromagnetic metamaterials, artificial optical materials with engineered optical properties beyond those which could be found in nature, are attracting increasing attention since 


\section{WILEY-VCH}

they offer a unique opportunity to observe new optical phenomena such as magnetism at high frequencies, negative refraction ${ }^{[21]}$ and superlensing, ${ }^{[22]}$ and open up opportunities for numerous applications in nanophotonics and nonlinear optics. ${ }^{[23-25]}$ Particularly, in contrast to ordinary media, a metamaterial with an artificially created hyperbolic dispersion, ${ }^{[26]}$ can support optical modes with theoretically infinitely high wavevectors and therefore provides extremely high local density of optical states, offering the opportunity to engineer spontaneous emission rates ${ }^{[27]}$ as well as improve resolution in optical imaging. ${ }^{[28]}$ Here, we show that coating of plasmonic nano-objects with a hyperbolic medium (Figure 1c,d) provides broad flexibility for designing their optical responses. The hyperbolic coating was experimentally demonstrated by coating of $\mathrm{Au}$ nanospheres, chosen as a classical plasmonic test object, with alternating $\mathrm{SiO}_{2}$ and $\mathrm{Au}$ multishells. We show that a variation on the parameters of the multishells allows for the easy creation and tuning of plasmonic modes covering a broad wavelength range from 400 to 2200 $\mathrm{nm}$. Theoretical calculations (in both multishell and effective medium descriptions) confirm the hyperbolic nature of the dispersion in the multishell coating, and further reveal that optical properties of plasmonic nanoparticles coated with a hyperbolic metamaterial, which we term "metaparticles", are highly dependent on the thickness of the coating, which can be tailored to a very large degree. The proposed concept was then extended to the hyperbolic coating of dielectric nanoparticles, further confirming the nature of the observed modes to be related to the resonances in the hyperbolic layer. These results suggest an attractive way for designing optical properties of nano-objects with high tunability, broadband response and strong localfield enhancement. It is also the first demonstration of colloidal version of hyperbolic metamaterials, which have advantages such as high flexibility in use (e.g., dispersed in solvents, spread on a surface, embedded in a solid medium) and easy excitation (e.g., by direct coupling with external illumination), and are interesting for a wide range of applications including spontaneous emission enhancement, enhanced spectroscopies, nanolasers, nonlinear optics, photothermal conversions, and hot-electron effects. 


\section{WILEY-VCH}

\section{Results}

\subsection{Experimental realization of hyperbolic coating}

The most common implementations of hyperbolic metamaterials are nanorod arrays or layered metal-dielectric structures. ${ }^{[23,25-27]}$ The latter method is particularly convenient for fabricating hyperbolic coating on various, possibly complex-shaped nano-objects. The hyperbolic coating of colloidal metallic nanoparticles (Figure 1c), which is a classical plasmonic object, can be realized by coating them with metal-dielectric multishells (Figure 1e) using a wet chemical method. ${ }^{[11,29]}$ When the multishell period is much smaller than the wavelength, the coating can be considered as an effective medium described by anisotropic Maxwell-Garnet theory. ${ }^{[30]}$ The permittivity tensor of such metamaterials is uniaxial in the spherical coordinate system, taking the form of $\varepsilon_{e f f}=\varepsilon_{r} \hat{r} \hat{r}+\varepsilon_{\theta} \hat{\theta} \hat{\theta}+\varepsilon_{\varphi} \hat{\varphi} \hat{\varphi}$, where $\varepsilon_{r}$ and $\varepsilon_{\theta}=\varepsilon_{\varphi}=\varepsilon_{t}$ are the components along and perpendicular to the radial direction. The real parts of $\varepsilon_{r}$ and $\varepsilon_{t}$ can have different signs (Figure 1f), resulting in the characteristic hyperbolic dispersion $k_{r}^{2} / \varepsilon_{t}+\left(k_{\theta}^{2}+k_{\varphi}^{2}\right) / \varepsilon_{r}=$ $\omega^{2} / c^{2}$ (shown in Figure 1d as an example), where $k_{r}, k_{\theta}$ and $k_{\varphi}$ are the components of the wavevectors of the electromagnetic wave propagating in the medium, $\omega$ is the frequency of the wave, and $c$ is the speed of light in vacuum.

To experimentally demonstrate the feasibility of such method, we fabricated metaparticles by alternatively coating 62-nm diameter $\mathrm{Au}$ nanospheres (Figure 2a) with $\mathrm{SiO}_{2}$ and $\mathrm{Au}$ shells (see Experimental Section). The $\mathrm{SiO}_{2}$ shell coating was realized using a modified Stöber process by controlled condensation of tetraethyl orthosilicate onto an Au surface in an alkaline environment, ${ }^{[31]}$ while the Au shell coating was formed using a chemical plating method by controlled reduction of $\mathrm{Au}$ ions onto an $\mathrm{Au}$-seed-functionalized $\mathrm{SiO}_{2}$ surface. ${ }^{[1]}$

Figure $2 b-d$ presents TEM images of metaparticles with one, two and three pairs of $\mathrm{SiO}_{2} / \mathrm{Au}$ shells respectively, showing good uniformity in size and shape. The onion-like multishell structures (schematic illustrations in Figure 2) were clearly revealed in the TEM 


\section{WILEY-VCH}

images, in which the $\mathrm{Au}$ and $\mathrm{SiO}_{2}$ shells can be distinguished with a very good intensity contrast (the $\mathrm{Au}$ shells are darker than the $\mathrm{SiO}_{2}$ shells) due to the difference in their compositions. The thicknesses of the $\mathrm{SiO}_{2}$ and $\mathrm{Au}$ shells $\left(t_{\mathrm{SiO}_{2}}\right.$ and $\left.t_{\mathrm{Au}}\right)$ were estimated to be about 10 and $21 \mathrm{~nm}$ respectively, and can be precisely controlled during the coating processes. The average diameters of metaparticles with one, two and three pairs of $\mathrm{SiO}_{2} / \mathrm{Au}$ shells are around 124, 186 and $248 \mathrm{~nm}$, respectively.

\subsection{Optical properties of metaparticles}

For Au nanospheres coated with an isotropic $\mathrm{SiO}_{2}$ shell (Figure $\mathrm{S} 2 \mathrm{a}-\mathrm{c}$, Supporting Information), the coating causes only a slight red shift in the dipolar resonance peak (Figure S2d, Supporting Information), which is due to the small contrast in the dielectric constants of silica and the surrounding medium (water), and the strong confinement of the electric field in the vicinity of Au nanospheres (Figure S2e,f, Supporting Information). However, Au nanospheres coated with anisotropic hyperbolic metamaterial present a rich, broadband and highly tunable plasmonic modal structure, as shown in Figure 3a-c. With an increasing number of $\mathrm{SiO}_{2} / \mathrm{Au}$ pairs, the optical properties of metaparticles change dramatically. The extinction spectrum of nanoparticles changed from having one resonance peak at $534 \mathrm{~nm}$ for uncoated Au nanospheres (Figure S2d, Supporting Information) to having two intense resonance peaks at 572 and 787 nm (Figure 3a) when the first pair of $\mathrm{SiO}_{2} / \mathrm{Au}$ shells was coated. Adding a second pair of $\mathrm{SiO}_{2} / \mathrm{Au}$ shells to the metaparticles induces an additional resonance around $1208 \mathrm{~nm}$ (Figure $3 \mathrm{~b}$ ), along with the red shift of the first peak (from 572 to $595 \mathrm{~nm}$ ) and the blue shift of the second peak (from 787 to $776 \mathrm{~nm}$ ). A similar behavior is observed for the metaparticles with three pairs of $\mathrm{SiO}_{2} / \mathrm{Au}$ shells (Figure 3c, black curve), with a further red shift of the first peak (from 595 to $614 \mathrm{~nm}$ ) and blue shift of the second peak (from 776 to $751 \mathrm{~nm}$ ). In parallel, the peak at $1208 \mathrm{~nm}$ observed for the metaparticles with two pairs of $\mathrm{SiO}_{2} / \mathrm{Au}$ shells blue-shifts to $1171 \mathrm{~nm}$ for the metaparticles with three pairs of $\mathrm{SiO}_{2} / \mathrm{Au}$ shells (Figure 3c, black curve). Moreover, increasing the coating from two to three pairs of $\mathrm{SiO}_{2} / \mathrm{Au}$ shells enables the 


\section{WILEY-VCH}

metaparticles to support two additional modes, at wavelengths of 875 and $1780 \mathrm{~nm}$, respectively

(Figure 3c). The mode at a longer wavelength is only observed when the metaparticles were transferred into chloroform (Figure 3c, red curve) to avoid strong absorption by water molecules in the near-infrared wavelength range. In addition to the broad resonance peaks, there were also several sharp peaks around 1409, 1912, 2022 and $2071 \mathrm{~nm}$ in the near-infrared wavelength range (Figure 3c, red curve). These sharp peaks are due to the surface-enhanced infrared absorption of thiolated polyethylene glycol molecules absorbed on the Au shells during the fabrication process (see Experimental Section), as confirmed by measuring an absorption spectrum of thiolated polyethylene glycol molecules (Figure S3, Supporting Information). This result indicates high local-field enhancement in the $\mathrm{SiO}_{2}$ shells between the Au shells, which will be discussed later. Dark-field scattering images and spectra of single metaparticles are presented in Figure S4 in the Supporting Information. The optical images clearly show welldispersed single metaparticles with color and intensity of which change dramatically with the increasing number of $\mathrm{SiO}_{2} / \mathrm{Au}$ pairs. The scattering spectra are in good agreement with the corresponding extinction spectra of metaparticles. The strong scattering of single metaparticles is promising for bio-imaging applications. It is worthy to mention that, compared to uncoated $\mathrm{Au}$ nanospheres or $\mathrm{Au}$ nanoshells, ${ }^{[32]}$ metaparticles with three pairs of $\mathrm{SiO}_{2} / \mathrm{Au}$ shells show greatly improved sensitivity (up to $740 \mathrm{~nm} /$ refractive index unit) to the refractive index changes in their surrounding (Figure S5, Supporting Information), which is attractive for optical sensing applications. In addition, the resonance modes of the metaparticles can also be tuned over a wide spectral range by controlling the thickness of Au shells (Figure S6, Supporting Information).

To get an insight into the experimental observations, finite element numerical simulations of scattering of an electromagnetic wave on metaparticles was performed, and the corresponding optical cross-sections were calculated (see Experimental Section). As shown in Figure $3 \mathrm{~d} 1, \mathrm{e} 1, \mathrm{f} 1$, using parameters of the experimentally studied structures shown in Figure 2, 


\section{WILEY-VCH}

the calculated extinction spectra of the metaparticles with one, two, and three pairs of $\mathrm{SiO}_{2} / \mathrm{Au}$ shells reproduce the experimental features very well (Figure $3 a-c)$. The much richer modal structure observed for thicker multishell coatings (Figure $3 \mathrm{e} 1, \mathrm{fl}$ ) is due to the fact that they support higher-order modes (see the modal analysis below). In order to confirm the metamaterial behavior, metaparticles with increased number of $\mathrm{SiO}_{2} / \mathrm{Au}$ pairs were also simulated by decreasing $t_{\mathrm{SiO}_{2}}$ and $t_{\mathrm{Au}}$ while keeping the total coating thickness unchanged, resulting in an increased shell density. Figure $3 \mathrm{~d} 2, \mathrm{e} 2, \mathrm{f} 2$ and $3 \mathrm{~d} 3, \mathrm{e} 3, \mathrm{f3}$ presents the optical spectra of metaparticles with the numbers of $\mathrm{SiO}_{2} / \mathrm{Au}$ pair two and four times of their original values (see schematics in the insets), respectively, but with the same effective permittivities. As can be clearly seen in Figure $3 \mathrm{~d} 1-\mathrm{d} 3,3 \mathrm{e} 1-\mathrm{e} 3$, and 3f1-f3, with the increase of the number of $\mathrm{SiO}_{2} / \mathrm{Au}$ pairs, when the total coating thickness is the same, the extinction spectra retain a similar shape with a general slight red shift of the peaks. Also it can be observed the appearance of new modes at longer wavelengths (compare Figure 3d2,d3; Figure 3e2,e3; Figure 3f2,f3 with Figure $3 \mathrm{~d} 1 ; 3 \mathrm{e} 1 ; 3 \mathrm{f} 1$, respectively), which is related to the extension of validity of hyperbolic dispersion to a higher wavevector region (see the discussion below) and the consequent support of higher-order modes in the multishell coating. Thus, the rich and broadband modal structure of the nanoparticles coated with a hyperbolic metamaterial can be engineered by the total thickness of the coating, while more precise tuning can be realized by controlling the number of $\mathrm{SiO}_{2} / \mathrm{Au}$ pairs with the fixed coating thickness.

\subsection{Effective medium description of metal-dielectric multishell coating}

To further understand the nature of optical effects related to the metal-dielectric multishell coating on a plasmonic nanoparticle, numerical simulations of scattering of an electromagnetic wave on $\mathrm{Au}$ nanospheres coated with a layer of homogeneous hyperbolic medium were performed and corresponding optical cross-sections were calculated (see Experimental Section). The permittivity of the latter was evaluated using an effective medium theory (EMT), considering the experimentally measured parameters of the studied structures (Figure 2). The 


\section{WILEY-VCH}

optical permittivity given by the EMT has a radial symmetry and possesses anisotropy, which can be expressed in a spherical coordinate system as $\varepsilon_{r}=\varepsilon_{\mathrm{Au}} \varepsilon_{\mathrm{SiO}_{2}} /\left(\left(1-f_{\mathrm{Au}}\right) \varepsilon_{\mathrm{Au}}+f_{\left.\mathrm{Au} \varepsilon_{\mathrm{SiO}_{2}}\right)}\right.$ and $\varepsilon_{\theta}=\varepsilon_{\varphi}=f_{A u} \varepsilon_{A u}+\left(1-f_{A u}\right) \varepsilon_{S_{i O}}$, where $f_{A u}=t_{A u} /\left(t_{A u}+t_{S i O_{2}}\right)$ is the Au filling factor, $\varepsilon_{A u}$ and $\varepsilon_{\mathrm{SiO}_{2}}$ are the permittivities of $\mathrm{Au}$ and $\mathrm{SiO}_{2}$. Above a certain wavelength, which depends on the filling factor, the permittivity components of the multishell coating have different signs (for the experimentally studied structures with $f_{A u}=0.68$, $\varepsilon_{\theta}$ and $\varepsilon_{\varphi}$ change signs from positive to negative at $250 \mathrm{~nm}$ (Figure 4a, beyond the measured wavelength range), for $f_{A u}=0.5$ at $490 \mathrm{~nm}$ (Figure S7a, Supporting Information) and for $f_{A u}=0.32$ at $530 \mathrm{~nm}$ (Figure S8a, Supporting Information)), leading to the hyperbolic dispersion of the metamaterial coating. Comparison of Figure $3 \mathrm{~d} 1-\mathrm{d} 3,3 \mathrm{e} 1-\mathrm{e} 3$ and $3 \mathrm{f} 1-\mathrm{f} 3$ with Figure $4 \mathrm{~b}, 4 \mathrm{c}$ and $4 \mathrm{~d}$ (calculated using the effective permittivities shown in Figure 4a), respectively, shows that with the increase of shell density (keeping the same filling factor), the coating becomes gradually better described by the EMT. Such tendency is related to a region of the EMT applicability: the EMT can correctly describe all the optical modes in the medium wavevectors of which are smaller than $\pi / \Lambda$, where $\Lambda$ is the multishell period. Thus, for the higher shell density (smaller $\Lambda$ ), the hyperbolic dispersion given by the EMT is valid for larger wavevectors in the dispersion presented in Figure 1d and can better describe the real structure. The agreement between the optical cross-sections given by the EMT and that of the multishell structure with the highest considered shell density is exceptionally good (compare Figure $4 b, 4 c$ and $4 d$ with Figure $3 d 3$, $3 \mathrm{e} 3$ and $3 \mathrm{f} 3$ ), confirming the hyperbolic nature of the dispersion in the metal-dielectric multishell coating. The above studied case of a hyperbolic coating of a nanoparticle which effectively creates a hyperbolic resonator formed by a metamaterial layer of a finite thickness, lays between the two extrema of a very thin hyperbolic coating and a scatterer embedded in an infinite hyperbolic medium. For thin coatings, a hyperbolic layer leads to a slight modification of the scattering properties of the nano-object, resulting in a minor red-shift of the dipolar 


\section{WILEY-VCH}

plasmonic resonance and a change in its magnitude (Figure S7, Supporting Information). In the case of thicker coating, approaching the limit of an infinite hyperbolic medium, the nano-object scatters light into a set of propagating modes supported by the hyperbolic medium, which can be expressed through Bessel functions and vector spherical harmonics. ${ }^{[30]}$

Further exploiting the opportunities given by the EMT approach, the impact of varying metal filling factors $f_{A u}(0.68$, studied above, 0.5 and 0.32$)$ on the modal structure was studied (Figure 4, Figure S8,9 in Supporting Information). It was found that optical properties of the hyperbolic medium coated Au nanospheres are quite robust to the filling factor parameter and, therefore, robust to the fabrication uncertainties of the shell thicknesses, defined mostly by the total thickness of the coating. This can be explained by the fact the electric fields of the modes are predominantly oriented in the radial direction, thus the modes are mostly sensitive to the radial dielectric permittivity component of the permittivity tensor, which only slightly changes with the filling factor.

\subsection{Near-field distributions}

In order to get a further insight into the nature of the observed optical resonances and identify them, spatial distributions of the electric field intensity $\left(|E|^{2} /\left|E_{0}\right|^{2}\right.$, where $E_{0}$ is the incident electric field) were plotted. In contrast to uncoated or silica-coated Au nanospheres (Figure S2e,f, Supporting Information), the metaparticles with three pairs of $\mathrm{SiO}_{2} / \mathrm{Au}$ shells possess both dipolar and quadrupolar resonances of various orders (the latter is related to the field distribution across the metamaterial layer). The nature of the short-wavelength resonance (Figure 5a1,b1,c1,d1) might be related to the excitation of bulk modes in the hyperbolic medium. Comparing the resonances of the same order, it was found that, as expected, the dipolar resonances are at the longer wavelengths than the quadrupolar ones (c.f., e.g., Figure 5a5,a6 with Figure 5a2,a3,a4, and also the corresponding plots in Figure 5b,c,d). If the same type of resonance is considered (e.g., dipolar), then the higher order resonances, both for the multilayered structures (with the field intensity localized in the further dielectric shell) and in 


\section{WILEY-VCH}

the EMT description, lie also at the longer wavelengths (Figure 5). A very good agreement between the intensity distributions of the resonances in the multishell structure and the one coated with the corresponding homogeneous hyperbolic medium was observed (becoming better with increasing shell density), which further confirms the feasibility of the realization of hyperbolic coating via metal-dielectric multishell coating.

The near-field distributions also allow evaluation of the electric field enhancement associated with different metaparticle resonances. As shown in Figure 5a, at the resonance conditions, the intensity of the electric fields in the multishell structure of metaparticles with three pairs of $\mathrm{SiO}_{2} / \mathrm{Au}$ shells is greatly enhanced. For example, the field intensity enhancements can reach the factors of $\sim 310,500$ and 220 for incident light at the wavelengths of 780,1200 , and $1800 \mathrm{~nm}$, respectively, which are much higher than the enhancement factor $(\sim 50)$ achievable in Au nanospheres (Figure S2e,f, Supporting Information). In addition, the intensity enhancement can be further increased to a maximum value of $\sim 3000$ by the increase of the shell density of the coating (Figure $5 b, c$ ), which is in a general agreement with higher field enhancement in narrower gaps in plasmonic nanostructures with the considered sizes, ${ }^{[33]}$ when the nonlocal effects are not yet important. These results explain the experimental observation of sharp surface-enhanced infrared absorption peaks in the extinction spectrum produced by thiolated polyethylene glycol molecules absorbed on the Au shells (Figure 3c). The high and spectrally broad local-field enhancement introduced by the hyperbolic coating of plasmonic nano-objects is extremely useful for applications such as enhanced infrared and Raman spectroscopies, ${ }^{[34]}$ nonlinear optics, ${ }^{[6]}$ hot-electron generation, ${ }^{[3,35]}$ and photo-thermal conversions. ${ }^{[36,37]}$

\subsection{Hyperbolic coatig of dielectric nano-objects}

Apart from the hyperbolic coating of plasmonic nano-objects, the concept was also extended to dielectric nano-objects (Figure 6a). Figure 6 shows optical cross-sections and near-field distributions of silica nanospheres coated with dielectric-metal mutishells and a corresponding 


\section{WILEY-VCH}

homogeneous hyperbolic medium (see also Figure S10 in the Supporting Infromation). A very good agreement between the two descriptions was shown, ascertaning the applicability of hyperbolic coating to modify optical responce of dielectric nano-objects. It is worth noting that in the case of a dielectric core, a response similar to the metallic core case was observed (Figure 3-5). This confirms that the nature of the modes and, therefore, the extinction spectrum of the metaparticle is essentially defined by the hyperbolic coating, acting as a resonator with very high density of local electromagnetic states.

\subsection{Applications}

The hyperbolic coating of nanoparitlces leads to the creation of a colloidal version of hyperbolic metamaterials. Compared with traditional hyperbolic optical metamaterials in the form of plasmonic nanorod assemblies or metal-dielectric multilayers fabricated on a subsrate, ${ }^{[23,25-27]}$ hyperbolic metaparticles shows many interesting advantages such as high flexibility in use (e.g., dispersed in a solvent, self-assembled on a surface or embedded in a bulk matrix) and easy excitation of hyperbolic modes (e.g., by direct scattering of external radiation). Combined with the properties demonstrated above, hyperbolic metaparticles are promising for a broad range of applications. The hyperbolic metaparticles can be used as antennas for the enhancement of spontaneous emission rate and outcoupling efficiency of embedded or external emitters ${ }^{[27,38-41]}$ by directly dispersing them into an active solution or spreading them on an active surface. It is also possible to make active hyperbolic metaparticles by incorporating optical gain (dye molecules or quantum dots) directly into the dielectric shells (Figure S11, Supporting Information), opening new opportunities for developing ultra-small single-particle nanolasers. ${ }^{[42]}$ In addition, single metaparticles can works as building blocks for the creation of complex hyperbolic structures via self assembly (Figure S12, Supporting Information) which are difficult to realize using exsiting fabrication methods.

\section{Conclusion}




\section{WILEY-VCH}

We have investigated experimentally and theoretically dressing of a plasmonic nano-object with an anisotropic hyperbolic coating, which allows engineering the refractive index beyond those available with natural materials, leading to the creation of colloidal form of hyperbolic metamaterials. The proposed approach provides an attractive way and an additional degree of freedom for designing an optical response of a nano-object, which can be easily extended to nanoparticles made from many other materials (e.g., other metals, semiconductors and dielectrics) and shapes (e.g., nanorods, nanocubes), or other complex nanostructures, ${ }^{[15]}$ with the possibility to obtain rich and interesting optical responses. Apart from the wet chemical method demonstrated above, the hyperbolic coating can also be realized in the form of dielectric/metallic multilayers by physical vapor deposition, ${ }^{[23,27]}$ which is especially useful for the coating of pre-designed nanostructures on a substrate. In addition, the coating is not limited to hyperbolic media, other anisotropic dielectric media or media with anisotropic permeability may also hold a great interest for the engineering of optical responses of nanophotonic objects.

\section{Experimental Section}

Fabrication of metaparticles: The metal-dielectric multishell coating was realized by alternately coating $\mathrm{Au}$ nanospheres with $\mathrm{SiO}_{2}$ and $\mathrm{Au}$ shells. The fabrication started with 62nm diameter $\mathrm{Au}$ nanospheres. The details for the $\mathrm{SiO}_{2}$ coating on nanoparticles with an $\mathrm{Au}$ outmost surface and Au coating on nanoparticles with a $\mathrm{SiO}_{2}$ outmost surface are as following:

1. $\mathrm{SiO}_{2}$ coating on nanoparticles with $\mathrm{Au}$ outmost surface. Before $\mathrm{SiO}_{2}$ coating, nanoparticles with an Au outmost surface were mixed with an aqueous solution of O-[2-(3Mercaptopropionylamino)ethyl]-O'-methylpolyethylene glycol (SH-PEG) to functionalize the $\mathrm{Au}$ surface with a monolayer of SH-PEG molecules. After purification with repeated centrifugations, nanoparticles were dispersed into $0.8 \mathrm{~mL}$ deionized (DI) water, and then mixed with $5 \mathrm{~mL}$ ethanol, $2 \mu \mathrm{L}$ tetraethyl orthosilicate, and $30 \mu \mathrm{L}$ ammonium hydroxide solution (28\% $\mathrm{NH}_{3}$ in $\mathrm{H}_{2} \mathrm{O}$ ). The resultant solution was stirred overnight and then washed three times with 


\section{WILEY-VCH}

ethanol. Figure $\mathrm{S} 1 \mathrm{a}, \mathrm{d}, \mathrm{g}$ in the Supporting Information shows TEM images of $\mathrm{SiO}_{2}$ coated $\mathrm{Au}$, $\mathrm{Au} /\left(\mathrm{SiO}_{2} / \mathrm{Au}\right)_{1}$ and $\mathrm{Au} /\left(\mathrm{SiO}_{2} / \mathrm{Au}\right)_{2}$ nanoparticles, respectively.

2. Au coating on nanoparticles with $\mathrm{SiO}_{2}$ outmost surface. The nanoparticles with a $\mathrm{SiO}_{2}$ outmost surface were first functionalized with 3-Aminopropyltrimethoxysilane (APTMS) for the subsequent attachment of $\mathrm{Au}$ seeds. Briefly, nanoparticles in ethanol were added with excessive APTMS under vigorous stirring and the solution was stirred overnight. Then, the solution was heated to $80^{\circ} \mathrm{C}$ and kept at this temperature for one hour to enhance the attachment of APTMS molecules on $\mathrm{SiO}_{2}$ surface and centrifuged four times to remove unreacted APTMS molecules.

2.1 Synthesis of Au seeds. Au seeds with 1-2 nm size were prepared using a modified method demonstrated by Duff et al. ${ }^{[43]}$ Briefly, under vigorous stirring, $\mathrm{NaOH}(0.5 \mathrm{~mL}, 1 \mathrm{M})$ and tetrakis(hydroxymethyl)phosphonium chloride solution $(1 \mathrm{~mL}, 68 \mathrm{mM})$ were added successively into $45 \mathrm{~mL}$ water. The resultant solution was stirred for further $5 \mathrm{~min}$. Then $\mathrm{HAuCl}_{4}(2 \mathrm{~mL}, 25 \mathrm{mM})$ was quickly added and the solution was stirred for another $30 \mathrm{~min}$. The obtained solution was aged at $4^{\circ} \mathrm{C}$ for at least two weeks before use.

2.2 Attachment of Au seeds to APTMS-functionalized $\mathrm{SiO}_{2}$ surface. In a $20 \mathrm{~mL}$ vial, $12 \mathrm{~mL}$ of Au seeds was added, followed by rapid addition of $\mathrm{HCl}(600 \mu \mathrm{L}, 0.1 \mathrm{M}), \mathrm{NaCl}(180 \mu \mathrm{L}, 1$ M), and APTMS-functionalized nanoparticles. The solution was quickly vortexed and sonicated for $15 \mathrm{~min}$. The resulting solution was incubated overnight, and centrifuged three times to remove free Au seeds and dispersed in DI water. Figure S1b,e,h in the Supporting Information shows TEM images of Au-seed-attached $\mathrm{Au} / \mathrm{SiO}_{2}, \mathrm{Au} /\left(\mathrm{SiO}_{2} / \mathrm{Au}\right)_{1} / \mathrm{SiO}_{2}$ and $\mathrm{Au} /\left(\mathrm{SiO}_{2} / \mathrm{Au}\right)_{2} / \mathrm{SiO}_{2}$ nanoparticles, respectively.

2.3 Growth of Au shell on $\mathrm{SiO}_{2}$ surface. The coating of an Au shell on the Au-seed-attached $\mathrm{SiO}_{2}$ surface was done using a plating solution as a source of $\mathrm{Au}^{3+}$. The plating solution was prepared by mixing $50 \mathrm{~mL}$ of water, $12.5 \mathrm{mg}$ of $\mathrm{K}_{2} \mathrm{CO}_{3}$, and $0.75 \mathrm{~mL}$ of $1 \mathrm{wt} \%$ aqueous $\mathrm{HAuCl}_{4}$ solution followed by aging at $4^{\circ} \mathrm{C}$ overnight. In a typical reaction, to a $20 \mathrm{~mL}$ vial, $2 \mathrm{~mL}$ plating 


\section{WILEY-VCH}

solution was added, followed by the addition of an appropriate amount of Au-seed-attached nanoparticle solution. Next, $10 \mu \mathrm{L}$ of formaldehyde solution $\left(36.5-38 \%\right.$ in $\mathrm{H}_{2} \mathrm{O}$ ) was quickly added, and the resultant solution was stirred for $15 \mathrm{~min}$. The thickness of the Au shell was controlled by altering the volume of Au-seed-attached nanoparticle solution in the reaction.

Finite element numerical modeling: Numerical simulations were performed using a finite element method (COMSOL Multiphysics). The scattering from Au nanospheres coated with metal-dielectric multishells as well as with homogeneous hyperbolic layer was studied in a scattering-field formulation with a simulation domain surrounded by a perfectly matched layer, to guarantee the absence of back-reflection from the outer domain boundaries. Dielectric constant for gold was taken from ref. 44 and extended to the infrared region using the derived Drude parameters, refractive indices for silica and surrounding water were taken as 1.45 and 1.33, respectively. The symmetry of the model was used to decrease the complexity of the simulations. The nanoparticle was placed in the centre of a spherical simulation domain, then all of the objects were cut by two planes both passing through the center of the model: one is the polarization plane of the incident linearly-polarized light and the other is perpendicular to it. Finally, only one quadrant of the space between these planes was used for the numerical evaluation with the appropriate boundary conditions set on its flat edge: perfect magnetic conductor for the polarization plane and perfect electric conductor for the other one. The validity of the scattering model was checked by benchmarking it against the results for scattering of a linearly polarized plane wave on a metallic nanoparticle from analytical simulations. $^{[45]}$ Extinction, scattering and absorption cross-sections (normalized to the corresponding geometrical cross-sections of the nanoparticles) were calculated using various and independent methods: direct integration of scattering field power flow over a surface surrounding the particle $\left(P_{\text {scat }}\right)$, direct integration of electromagnetic losses over the nanoparticle volume $\left(W_{a b s}\right)$ and power flow entering it $\left(P_{\text {part }}\right)$, as well as the optical theorem $\left(P_{O T}\right)\left(\right.$ Ext1: $P_{O T}$, Ext2: $P_{\text {part }}+P_{\text {scat }}$, Scat $1: P_{O T}-W_{a b s}$, Scat2: $P_{\text {scat }}$, Abs $1: W_{a b s}$, Abs2: 


\section{WILEY-VCH}

$\left.P_{\text {part }}\right)$. The results obtained from the different methods were found to be in excellent agreement, which further confirms the validity of the model and the calculation techniques. The field maps were reconstructed over the entire space again using the symmetry of the model.

\section{Supporting Information}

Supporting Information is available from the Wiley Online Library or from the author.

\section{Acknowledgements}

This work has been funded in part by the Engineering and Physical Sciences Research Council (UK) and the European Research Council iPLASMM project (321268). A.V.Z. acknowledges support from the Royal Society and the Wolfson Foundation. Pan Wang and Alexey V. Krasavin contributed equally to this work.

Received: ((will be filled in by the editorial staff))

Revised: ((will be filled in by the editorial staff)) Published online: ((will be filled in by the editorial staff)) 


\section{WILEY-VCH}

References

1. Gramotnev, D. K. \& Bozhevolnyi, S. I. Nat. Photon. 4, 83-91 (2010).

2. Ciracì, C. et al. Science 337, 1072-1074 (2012).

3. Brongersma, M. L., Halas, N. J. \& Nordlander, P. Nat. Nanotechnol. 10, 25-34 (2015).

4. Kinkhabwala, A. et al. Nat. Photon. 3, 654-657 (2009).

5. Akselrod, G. M. et al. Nat. Photon. 8, 835-840 (2014).

6. Kauranen, M. \& Zayats, A. V. Nat. Photon. 6, 737-748 (2012).

7. Sherry, L. J. et al. Nano Lett. 5, 2034-2038 (2005).

8. Liu, N., Tang, M. L., Hentschel, M., Giessen, H. \& Alivisatos, A. P. Nat. Mater. 10, 631$636(2011)$.

9. Atwater, H. A. \& Polman, A. Nat. Mater. 9, 205-213 (2010).

10. Wu, K., Chen, J., McBride, J. R. \& Lian, T. Science 349, 632-635 (2015).

11. Oldenburg, S. J., Averitt, R. D., Westcott, S. L. \& Halas, N. J. Chem. Phys. Lett. 288, 243247 (1998).

12. Angelis, C. D., Locatelli, A., Modotto, D., Boscolo, S., Midrio, M. \& Capobianco, A. J. Opt. Soc. Am. B 27, 997-1001 (2010).

13. Radloff, C. \& Halas, N. J. Nano Lett. 4, 1323-1327 (2004).

14. Chen, H. J., Shao, L., Li, Q. \& Wang, J. F. Chem. Soc. Rev. 42, 2679-2724 (2013).

15. Fan, J. A. et al. Science 328, 1135-1138 (2010).

16. Zhou, W. \& Odom, T. W. Nat. Nanotechnol. 6, 423-427 (2011).

17. Lei, D. Y. et al. ACS Nano 6, 1380-1386 (2012).

18. Schmidt, M. A., Lei, D. Y., Wondraczek, L., Nazabal, V. \& Maier, S. A. Nat. Commun. 3, 1108 (2012).

19. Wang, P. et al. Nano Lett. 15, 7581-7586 (2015).

20. Lu, Y., Yin, Y. D., Li, Z. Y. \& Xia, Y. N. Nano Lett. 2, 785-788 (2002).

21. Smith, D. R., Pendry, J. B. \& Wiltshire, M. C. K. Science 305, 788-792 (2004). 


\section{WILEY-VCH}

22. Pendry, J. B. Phys. Rev. Lett. 85, 3966-3969 (2000).

23. Yang, X. D., Yao, J., Rho, J., Yin, X. B. \& Zhang, X. Nat. Photon. 6, 450-454 (2012).

24. Hess, O. et al. Nat. Mater. 11, 573-584 (2012).

25. Nicholls, L. H. et al. Nat. Photon. 11, 628-633 (2017).

26. Poddubny, A., Iorsh, I., Belov, P. \& Kivshar, Y. Nat. Photon. 7, 958-967 (2013).

27. Lu, D., Kan, J. J., Fullerton, E. E. \& Liu, Z. W. Nat. Nanotechnol. 9, 48-53 (2014).

28. Liu, Z. W., Lee, H., Xiong, Y., Sun, C. \& Zhang, X. Science 315, 1686 (2007).

29. Prodan, E., Radloff, C., Halas, N. J. \& Nordlander, P. Science 302, 419-422 (2003).

30. Wu, C., Salandrino, A., Ni, X. J. \& Zhang, X. Phys. Rev. X4, 021015 (2014).

31. Stöber, W., Fink, A. \& Bohn, E. J. Colloid Interface Sci. 26, 62-69 (1968).

32. Lal, S., Link, S. \& Halas, N. J. Nat. Photon. 1, 641-648 (2007).

33. Xu, H. X. Phys. Rev. B 72, 073405 (2005).

34. Lim, D. -K., Jeon, K. -S., Kim, H. M., Nam, J. -M. \& Suh, Y. D. Nat. Mater. 9, 60-67 (2010).

35. Wang, P., Krasavin, A. V., Nasir, M. E., Dickson, W. \& Zayats, A. V. Nat. Nanotechnol. 13, 159-164 (2018).

36. Baffou, G. \& Quidant, R. Laser Photonics Rev. 7, 171-187 (2013).

37. Meng, L. J., Yu, R. W., Qiu, M. \& García de Abajo, F. J. ACS Nano 11, 7915-7924 (2017).

38. Guclu, C., Luk, T. S., Wang, G. T. \& Capolino, F. Appl. Phys. Lett. 105, 123101 (2014).

39. Slobozhanyuk, A. P. et al. Phys. Rev. B 92, 195127 (2015).

40. Lu, D. et al. Adv. Mater. 30, 1706411 (2018).

41. Ayala-Orozco, C. et al. Nano Lett. 14, 2926-2933 (2014).

42. Wan, M. J., Gu, P., Liu, W. Y., Chen, Z. \& Wang, Z. L. Appl. Phys. Lett. 110, 031103 (2017).

43. Duff, D. G. \& Baiker, A. Langmuir 9, 2301-2309 (1993).

44. Johnson, P. B. \& Christy, R. W. Phys. Rev. B 6, 4370-4379 (1972).

45. Parsons, J., Burrows, C. P., Sambles, J. R. \& Barnes, W. L. J. Mod. Opt. 57, 356-365 (2010). 


\section{WILEY-VCH}

a

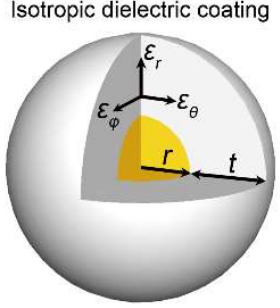

b

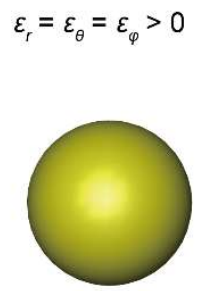

c

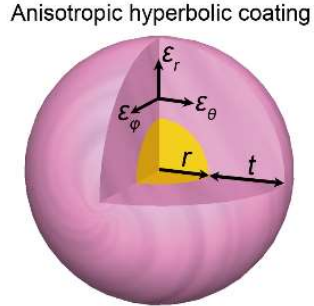

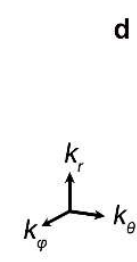

$\varepsilon_{r}>0, \varepsilon_{\theta}=\varepsilon_{\varphi}<0$

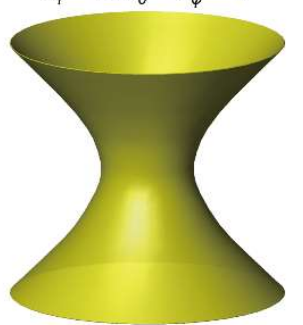

e Experimental realization

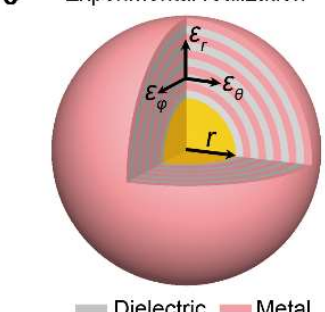

f

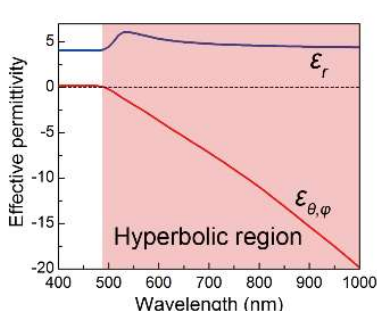

Figure 1. Isotropic dielectric coating versus anisotropic hyperbolic coating. a,c) Schematics of a plasmonic nanosphere coated with an isotropic dielectric medium (a) and a homogeneous anisotropic hyperbolic medium (c), where $r$ is the radius of the nanosphere, $t$ is the thickness of the coating. b,d) Isofrequency surfaces for the isotropic dielectric coating in (a) $\left(\varepsilon_{r}=\varepsilon_{\theta}=\right.$ $\varepsilon_{\varphi}>0$ ) (b) and the anisotropic hyperbolic coating in (c) $\left(\varepsilon_{r}>0, \varepsilon_{\theta}=\varepsilon_{\varphi}<0\right)(\mathrm{d})$. e) Experimental realization of the hyperbolic coating in (c) with a metal-dielectric multishell coating. f) Real parts of effective permittivity of the metal-dielectric multishells in (e) determined with the effective media theory which gives a hyperbolic dispersion in the shaded wavelength range. As an example, the metallic and dielectric shells are considered as Au and $\mathrm{SiO}_{2}$ shells with the same thickness. 


\section{WILEY-VCH}
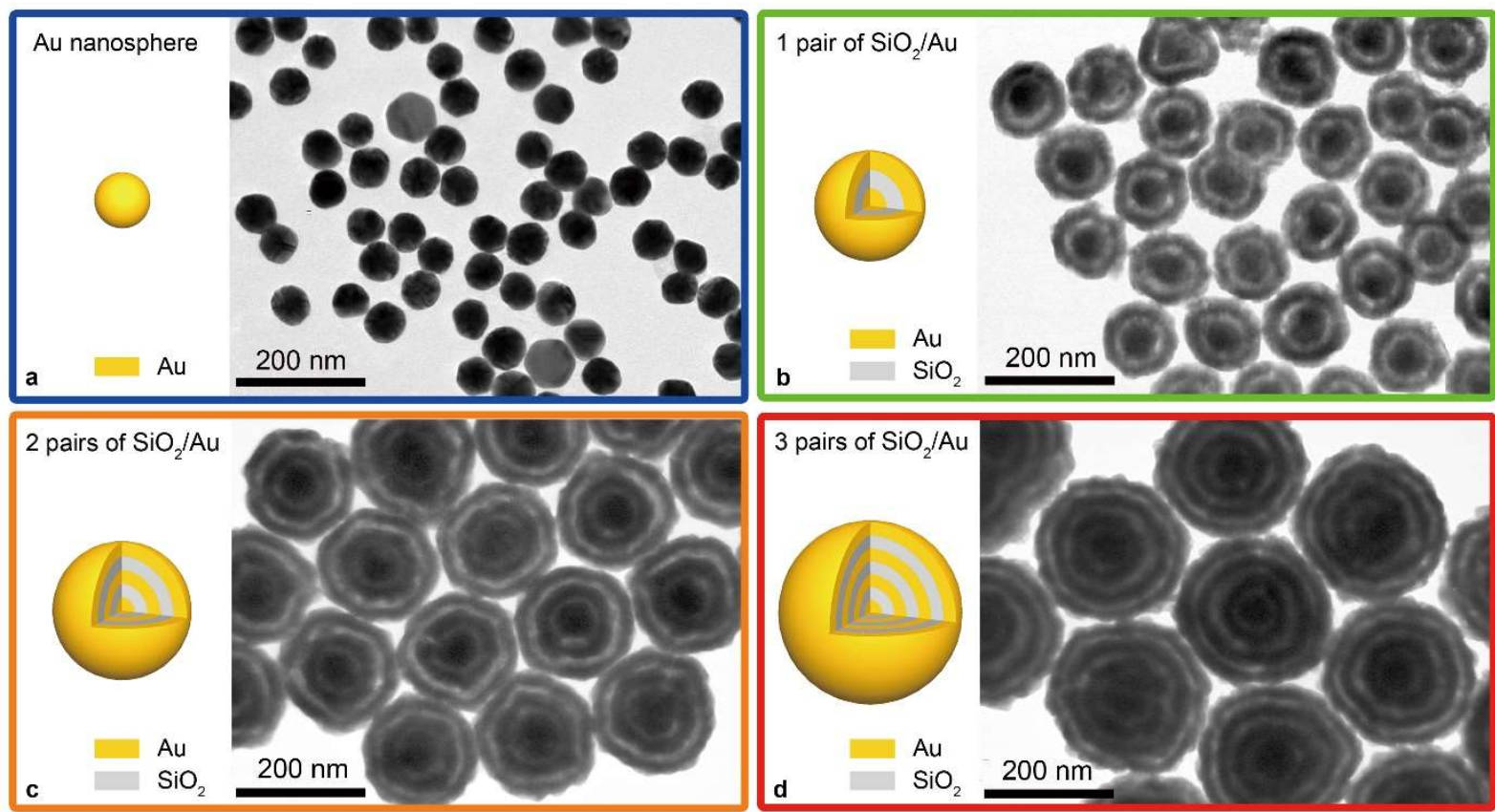

Figure 2. TEM images of uncoated Au nanospheres (a) and metaparticles with one (b), two (c) and three (d) pairs of $\mathrm{SiO}_{2} / \mathrm{Au}$ shells, respectively. The average diameter of $\mathrm{Au}$ nanospheres in (a) is $\sim 62 \mathrm{~nm}$, and the average thicknesses of $\mathrm{SiO}_{2}$ and $\mathrm{Au}$ shells in (b-d) are $\sim 10$ and $21 \mathrm{~nm}$, respectively. Insets: corresponding schematic illustrations. 
WILEY-VCH
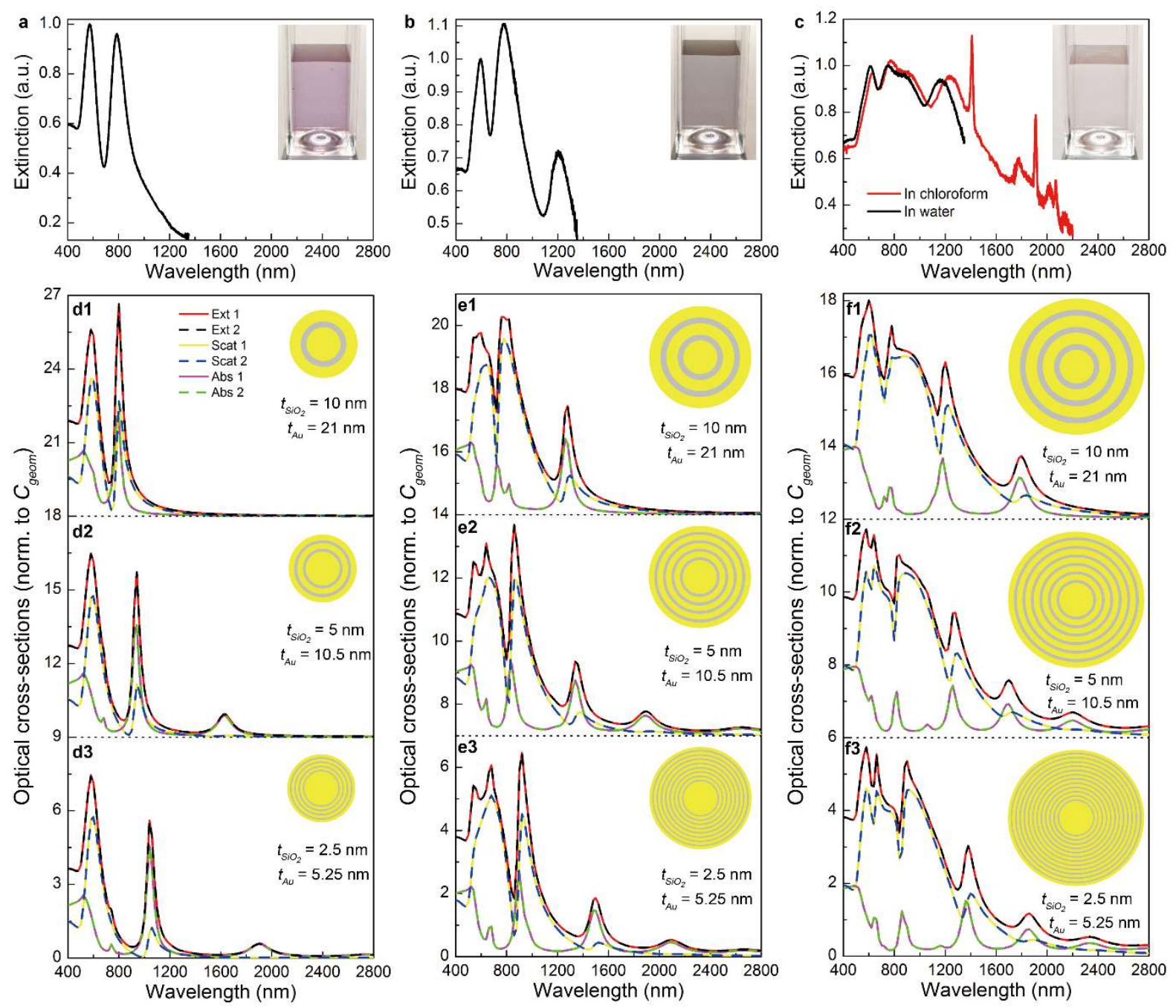

Figure 3. Optical properties of metaparticles. a-c) Experimentally measured extinction spectra of metaparticles with one (a) and two (b) pairs of $\mathrm{SiO}_{2} / \mathrm{Au}$ shells dispersed in water, and three (c) pairs of $\mathrm{SiO}_{2} / \mathrm{Au}$ shells dispersed in water (black curve) and chloroform (red curve). Insets: corresponding photos of the aqueous solutions of the metaparticles. d-f) Numerical simulation results: calculated optical (absorption, scattering and extinction) cross-sections, normalized to the corresponding geometrical cross-sections $\left(C_{g e o m}=\pi(r+t)^{2}\right)$, of the metaparticles with an overall shell thickness $(t)$ of $31 \mathrm{~nm}(\mathrm{~d} 1-\mathrm{d} 3), 62 \mathrm{~nm}(\mathrm{e} 1-\mathrm{e} 3)$ and $93 \mathrm{~nm}(\mathrm{fl}-\mathrm{f} 3)$, respectively. Corresponding schematics of the calculated structures and the thicknesses of silica $\left(t_{\mathrm{SiO}_{2}}\right)$ and Au shells $\left(t_{A u}\right)$ are shown in the insets. Curve label in (d) is applicable to (e) and (f). The optical cross-sections (Ext1, Ext2, Scat1, Scat2, Abs1, Abs2) were calculated using various approaches detailed in Experimental Section. 
WILEY-VCH
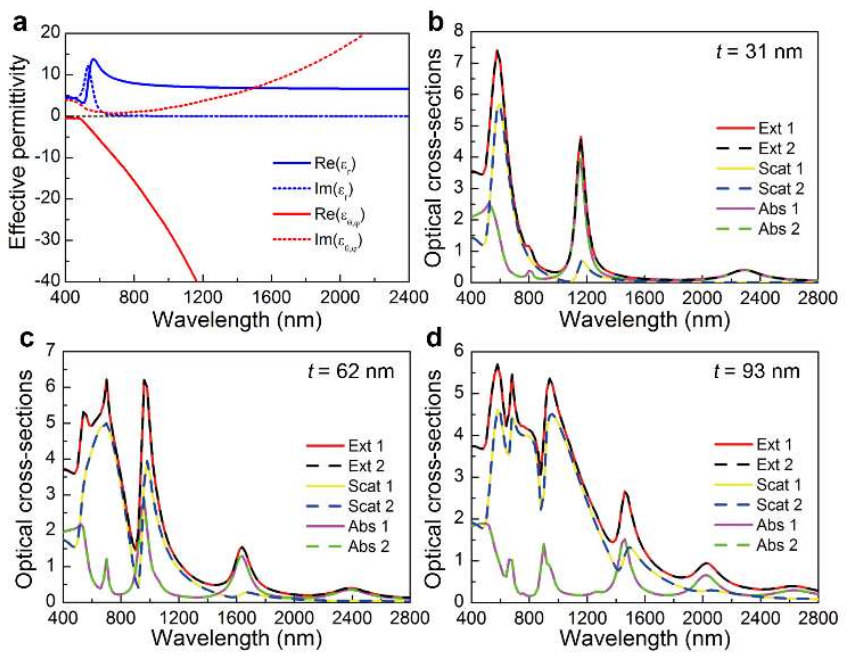

Figure 4. Optical properties of $\mathrm{Au}$ nanospheres with homogeneous hyperbolic coating. a) Effective permittivity determined using the EMT of $\mathrm{SiO}_{2} / \mathrm{Au}$ multishells with $\mathrm{Au}$ filling factor $\left(f_{\mathrm{Au}}\right)$ of $\left.0.68\left(t_{\mathrm{SiO}_{2}}=10 \mathrm{~nm}, t_{\mathrm{Au}}=21 \mathrm{~nm}\right) . \mathrm{b}-\mathrm{d}\right)$ Numerical simulation results: calculated optical (absorption, scattering and extinction) cross-sections, normalized to the corresponding geometrical cross-sections $\left(C_{g e o m}=\pi(r+t)^{2}\right)$, of Au nanospheres with a homogeneous hyperbolic coating layer with permittivity shown in (a) for coating thicknesses $(t): 31 \mathrm{~nm}(\mathrm{~b})$, $62 \mathrm{~nm}(\mathrm{c})$ and $93 \mathrm{~nm}(\mathrm{~d})$. 


\section{WILEY-VCH}
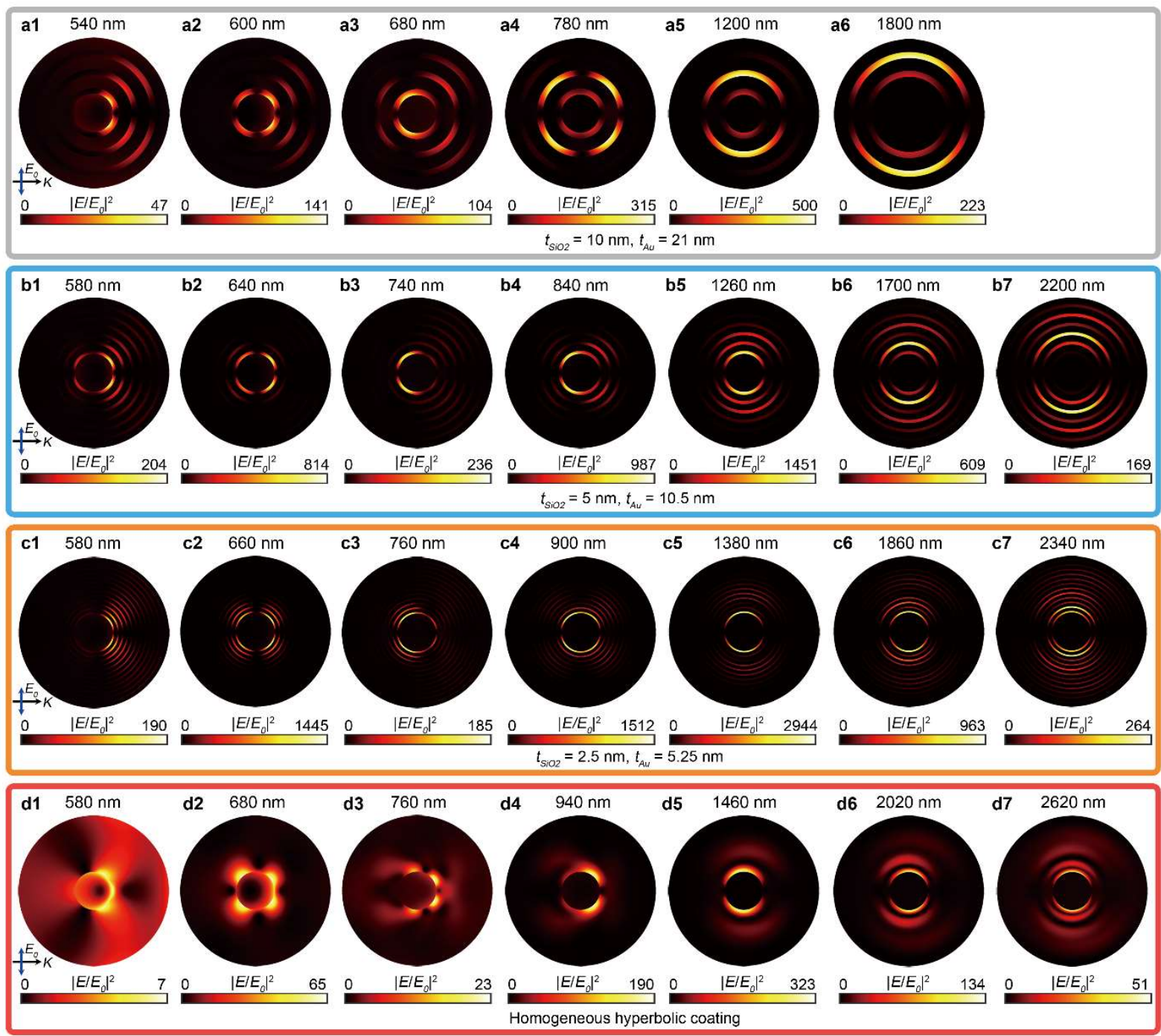

Figure 5. Electric field distributions of metaparticles. a-c) Electric field distributions for a metaparticle with three pairs of $\mathrm{SiO}_{2} / \mathrm{Au}$ shells $\left(t_{\mathrm{SiO}_{2}}=10 \mathrm{~nm}, t_{\mathrm{Au}}=21 \mathrm{~nm}\right)$ plotted at the wavelengths corresponding to the resonance peaks shown in Figure $3 \mathrm{fl}(\mathrm{a})$, six pairs of $\mathrm{SiO}_{2} / \mathrm{Au}$ shells with two times smaller shell thicknesses $\left(t_{\mathrm{SiO}_{2}}=5 \mathrm{~nm}, t_{\mathrm{Au}}=10.5 \mathrm{~nm}\right)$ plotted at the wavelengths corresponding to the resonance peaks shown in Figure $3 \mathrm{f} 2$ (b), and twelve pairs of $\mathrm{SiO}_{2} / \mathrm{Au}$ shells with four times smaller thicknesses $\left(t_{\mathrm{SiO}_{2}}=2.5 \mathrm{~nm}, t_{\mathrm{Au}}=5.25 \mathrm{~nm}\right)$ plotted at the wavelengths corresponding to the resonance peaks shown in Figure $3 \mathrm{f3}$ (c). d) Electric field distributions for an Au nanosphere coated with a layer of homogeneous hyperbolic medium with the thickness and EMT permittivity corresponding to all the designs considered above, plotted at the wavelengths corresponding to the resonance peaks shown in Figure 4d. 


\section{WILEY-VCH}
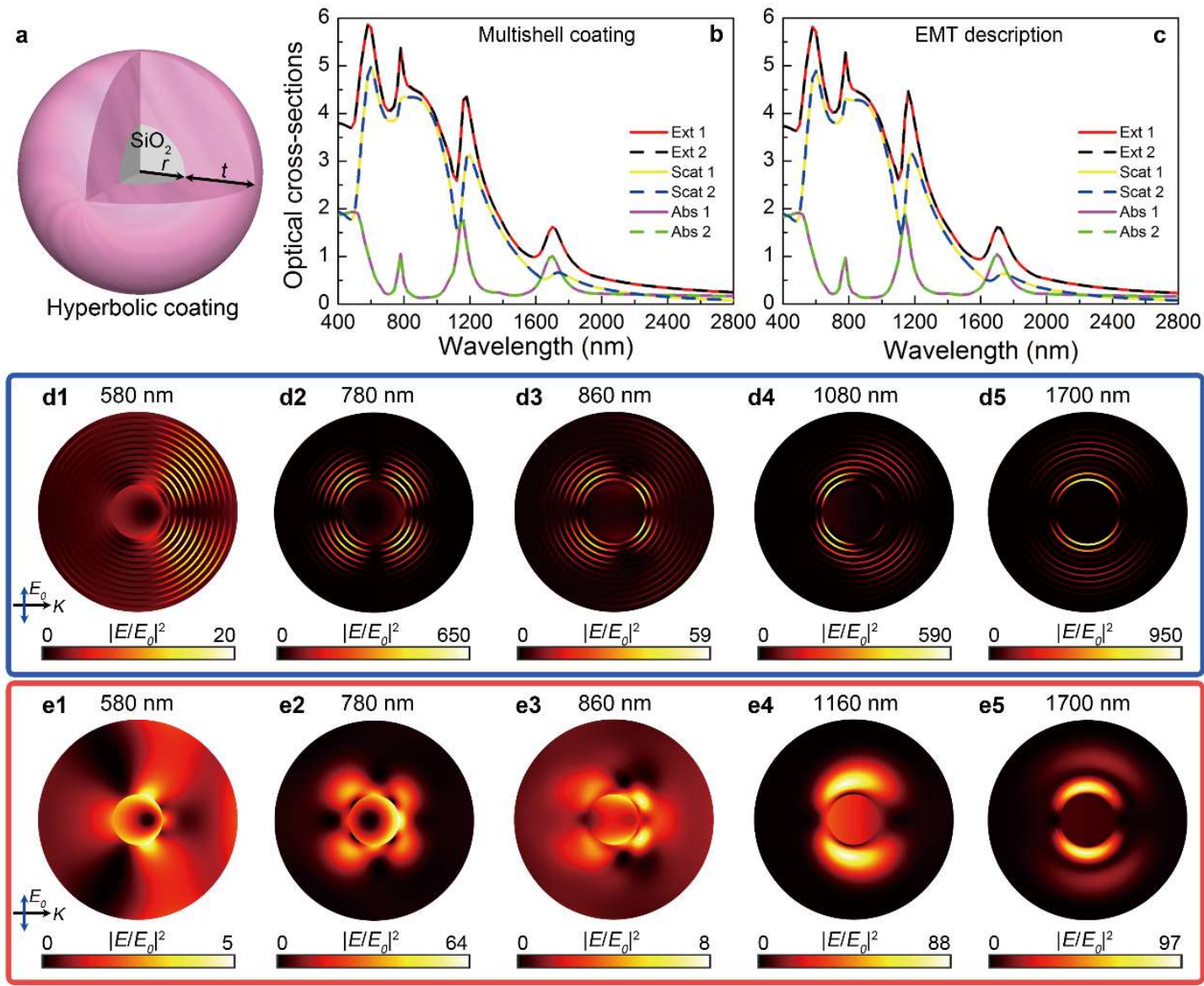

Figure 6. a) Schematic of a $\mathrm{SiO}_{2}$ nanosphere coated with a hyperbolic medium. b,c) Calculated optical (absorption, scattering and extinction) cross-sections, normalized to the corresponding geometrical cross-sections $\left(C_{\text {geom }}=\pi(r+t)^{2}\right)$, of a $\mathrm{SiO}_{2}$ nanosphere $(r=31 \mathrm{~nm})$ with twelve pairs of $\mathrm{SiO}_{2} / \mathrm{Au}$ shells $\left(t_{\mathrm{SiO}_{2}}=2.5 \mathrm{~nm}, t_{\mathrm{Au}}=5.25 \mathrm{~nm}\right)(\mathrm{b})$ and a homogeneous hyperbolic coating layer with permittivity shown in Figure 4a for a coating thickness $t$ of $93 \mathrm{~nm}(\mathrm{c})$. d,e) Electric field distributions for $\mathrm{SiO}_{2}$ nanosphere with twelve pairs of $\mathrm{SiO}_{2} / \mathrm{Au}$ shells (d) and a layer of homogeneous hyperbolic medium (e) plotted at the wavelengths corresponding to the resonance peaks shown in (b) and (c), respectively. 


\section{WILEY-VCH}

The table of contents entry:

The concept of coating nano-objects with an anisotropic metamaterial, which displays a hyperbolic dispersion and allows the design of refractive index on demand, is reported. This is experimentally demonstrated by coating $\mathrm{Au}$ nanospheres with alternating $\mathrm{SiO}_{2}$ and $\mathrm{Au}$ multishells, which creates rich and highly tuneable plasmonic modal structures covering a broad wavelength range and produces highly enhanced local fields.

Keywords: metaparticles, hyperbolic metamaterials, plasmonics, multishells, coating Pan Wang*, Alexey V. Krasavin, Francesco N. Viscomi, Ali M. Adawi, Jean-Sebastien Bouillard, Lei Zhang, Diane J. Roth, Limin Tong, Anatoly V. Zayats*

Metaparticles: dressing nano-objects with a hyperbolic coating

\section{ToC figure}

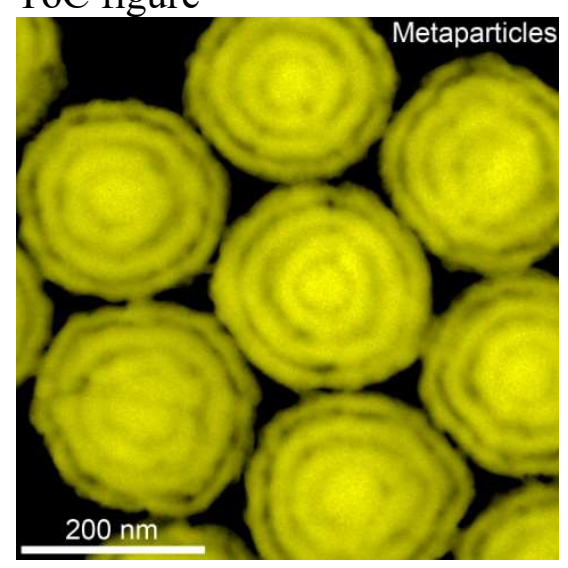

